\title{
Surface Modification of Aramid Fibres with Graphene Oxide for Interface Improvement in Composites
}

\author{
Lei Zeng ${ }^{1}$ Xuqing Liu ${ }^{1} \cdot$ Xiaogang Chen ${ }^{1}$. \\ Constantinos Soutis ${ }^{1,2}$
}

Received: 8 May 2018 / Accepted: 26 June 2018 / Published online: 6 August 2018

(C) The Author(s) 2018

\begin{abstract}
A novel method of biomimetic surface modification was used for aramid fibres aiming to enhance the interface properties between epoxy resin and the modified aramid fibre. Inspired by the composition of adhesive proteins in mussels, a thin layer of poly(dopamine) (PDA) was self-polymerized onto the surface of the aramid fibre. The graphene oxide (GO) was then grafted on the surface of PDA-coated aramid fibres. The microstructure and chemical characteristics of the pristine and modified fibres were characterised using Scanning Electron Microscopy (SEM) and X-ray photoelectron spectroscopy (XPS), indicating successful grafting of GO on the PDA-coated aramid fibres. Single fibre tensile test and microbond test were carried out to evaluate the mechanical properties of the modified fibres. It was found that the fibre surface modification improved the interfacial shear strength by $210 \%$ and the fibre tensile strength was protected by GO-PDA coating.
\end{abstract}

Keywords Surface modification · Graphene oxide · Interface · Aramid fibre composites · Epoxy resin

\section{Introduction}

Fibre reinforced composites have been widely used in aerospace, automobile, and military industries, due to their high specific strength, modulus, lightweight and flexible design. Particularly, aramid fibre is an ideal reinforcement for high-performance composites as it contains high mechanical properties, chemical stability and low density [1].

Xiaogang Chen

xiaogang_chen@hotmail.com

1 School of Materials, The University of Manchester, Manchester M13 9PL, UK

2 Aerospace Research Institute, The University of Manchester, Manchester, UK 
The surface of aramid fibres is smooth and chemically inert which lead to less strong interface with the matrix. It has been reported that weak bonding can lead to low load transfer efficiency between the fibre and resin in a fibre composite [2]. To enhance the interfacial adhesion strength of aramid fibre-reinforced composites, efforts were mainly made to modify fibre surface using plasma treatment, $\gamma$-ray irradiation, ultrasonic treatment, chemical grafting and etching [3-5]. However, these techniques request multistep procedures and are associated to the use of high cost instrument. In addition, these surface treatments are limited with strict reaction condition and in some case the use of toxic materials. Due to the damage caused to the fibre surface, fibre strength reduction is inevitable.

This paper aims to improve the interfacial adhesion between aramid fibres and epoxy resin based on the use of a novel method for fibre surface treatment at ambient temperature. A biopolymer, polydopamine (PDA), is applied to form a coating layer on the surface of fibre via $\mathrm{pH}$-induced oxidative self-polymerization, introducing functional groups such as carboxyl, amine and catechol for bonding enhancing between the fibre and resin to improve the physical properties of the composites [6, 7]. Then, graphene oxide (GO) is to be grafted onto the polydopamine-treated aramid fibre to enhance the mechanical property of the aramid fibre and to improve the interfacial bonding between fibre and resin. As a 2D material, GO has high aspect ratio with flexible structure, and it also has extraordinary mechanical properties and superior thermal and electrical conductivity. Graphene oxide is chemically more active with the fibre and resin polymers than graphene, because of the epoxide and hydroxyl groups within the GO sheets and carbonyl and carboxyl at the edges of the GO sheets. Evaluations of the coated aramid fibre are to be tested for strength and for interface adhesion with the epoxy resin.

\section{Experiment Details}

\subsection{Materials}

The aramid fibres used in this research were type 248 from Bluestar (China). The aramid fibres were rinsed by acetone to remove surface sizing. Dopamine hydrochloride, epoxy (AY103) and hardener (HY951) were purchased from Sigma-Aldrich ${ }^{\circledR}$ (UK). The Tris(hydoxymethy)aminomethane (Tris) was purchased from Alfa Aesar Company (USA). The Hydrochloric acid solution was purchased from Fluka Analytical (UK) and used as received. Graphene oxide water dispersion (TM-01LI-06) was purchased from Institute of Coal Chemistry, Chinese Academy of Sciences (China).

\subsection{Surface Functionalization of Aramid Fibres}

Dopamine solution $(2 \mathrm{mg} / \mathrm{mL}$ ) was prepared by dissolving dopamine in Tris- $\mathrm{HCl}$ buffer solution $(1.2 \mathrm{mg} / \mathrm{mL})$ and the $\mathrm{pH}$ was controlled as 8.5 . When dopamine was self-oxidising, the aramid fibre bundles were immersed in the solution at ambient temperature for $24 \mathrm{~h}$ to prepare the PDA-aramid fibre. For the GO-PDA-aramid fibre, after mixing dopamine with Tris- $\mathrm{HCl}$ buffer solution, aramid fibre bundles were immersed and $0.1 \mathrm{mg} / \mathrm{mL}$ GO solution was added into the mixture. The $\mathrm{pH}$ was 
controlled as 8.5. After $24 \mathrm{~h}$, aramid fibres were taken out of the solution and rinsed by distilled water, and then dried in oven at $60^{\circ} \mathrm{C}$. The schematic of process was shown in the Fig. 1.

\subsection{Characterization of Aramid Fibres}

The UV-visible spectra of dopamine in solution were recorded on UV-Visible spectrophotometer (M550 Double Beam Scanning UV/Visible spectrophotometer, Camspec Ltd., UK) using $\mathrm{H}_{2} \mathrm{O}$ as solvent. The scan range was 200-600 nm. All measurements were performed in quartz cuvettes.

The morphology of untreated and treated aramid fibres were studied by a Scanning Electron Microscopy SEM (Ultra-55 Scanning Electron Microscope, ZEISS, Germany). The aramid fibre samples were sputter-coated with gold before observation.

The chemical composition and functional groups on untreated and treated aramid fibre were characterised by X-ray Photoelectron Spectroscopy XPS (Kratos Axis Ultra Hybrid, Shimadzu, Japan) equipped with a monochromatic source of $\mathrm{Al} \mathrm{K} \alpha$, base pressure below $5 \times 10^{-8} \mathrm{mbar}$, spot area of $300 \mu \mathrm{m} \times 700 \mu \mathrm{m}$. The binding energy peaks were calibrated with $\mathrm{C} 1 \mathrm{~s}$ at $284.8 \mathrm{eV}$ as reference.

a

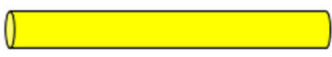

Aramid fibre

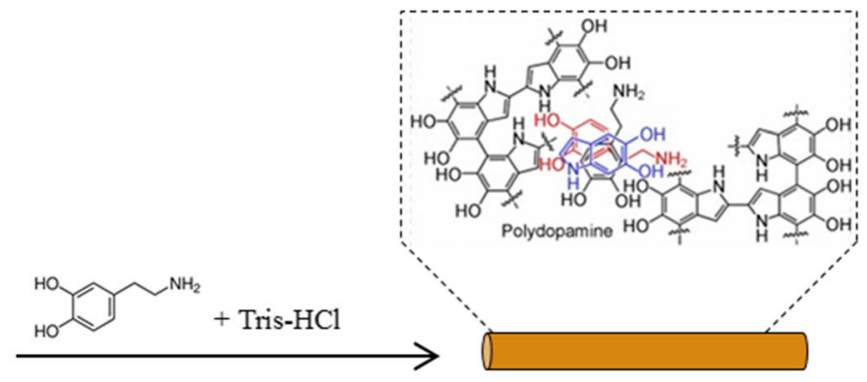

Ambient temperature $\mathrm{pH}=8.5$
PDA-aramid fibre

b

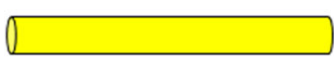

Aramid fibre

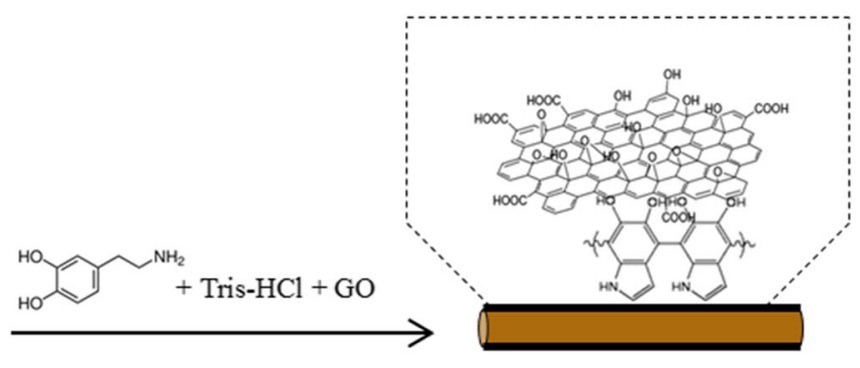

GO-PDA-aramid fibre $\mathrm{pH}=8.5$

Fig. 1 Schematic of (a) PDA-treated aramid fibre and (b) GO-PDA-treated aramid fibre preparation process 


\subsection{Mechanical Property Testing of Composites}

A microbond test was designed as depicted in Fig. 2 for the purpose of quantitatively identifying the interfacial properties between aramid fibres and resin matrix.

The interfacial shear strength (IFSS) between a single fibre and resin is given by Eq. (1):

$$
\mathrm{IFSS}=\frac{\mathrm{F}}{\pi \mathrm{d}_{\mathrm{f}} \mathrm{L}_{\mathrm{e}}}
$$

where $F$ is the failure tensile load, $d_{f}$ is the fibre diameter and $L_{e}$ is the embedded length in the resin. 50 data were collected and average IFSS was calculated for each sample.

The single fibre tensile test was conducted on Instron 1122 with a $5 \mathrm{~N}$ load cell at a constant speed of $1 \mathrm{~mm} \mathrm{~min}^{-1} .10$ specimens of each type of fibres were tested.

\section{Results and Discussion}

\subsection{Self-Polymerisation of Polydopamine}

The polymerisation process of dopamine in solution was detected and confirmed by UVvisible spectroscopy using $\mathrm{H}_{2} \mathrm{O}$ as solvent and the results are shown in Fig. 3. From the UVvisible spectrum, an absorption band at $405 \mathrm{~nm}$ due to the formation of quinone is clearly shown, which is in agreement with the mechanism of polymerisation. A typical spectrum of polydopamine is obtained in the supernatant after $24 \mathrm{~h}$. The results of $\mathrm{UV}$-visible spectroscopy showed three main absorbance peaks, the sharp absorbance peaks at $265 \mathrm{~nm}$ (catechol group), shoulder peaks at $300 \mathrm{~nm}$ (dehydro-dopamine), and wide peaks at $405 \mathrm{~nm}$ (o-quinone). The intensity of these groups was observed to increase over time, which suggests the formation of various intermediates [8]. The photo in Fig. 3 demonstrates the colour change of the solution (from clear to light brown within $5 \mathrm{~min}$, then dark brown after $24 \mathrm{~h}$ ) during PDA formation.

\subsection{Surface Chemical Structure of Aramid Fibre}

The chemical compositions of aramid fibre surfaces were studied by X-ray photoelectron spectroscopy (XPS) and the fitting of C1s core level spectra results are presented in Table 1.

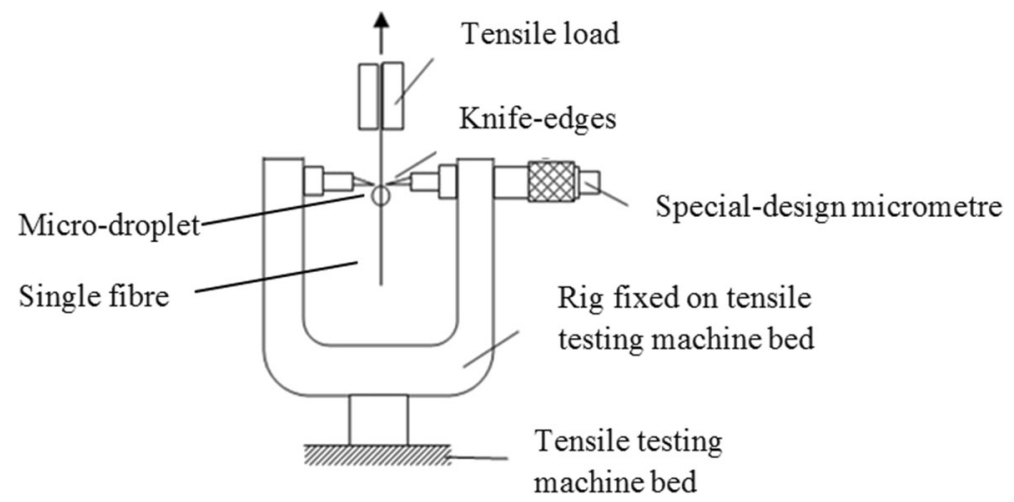

Fig. 2 Schematic of microbond test machine setting 


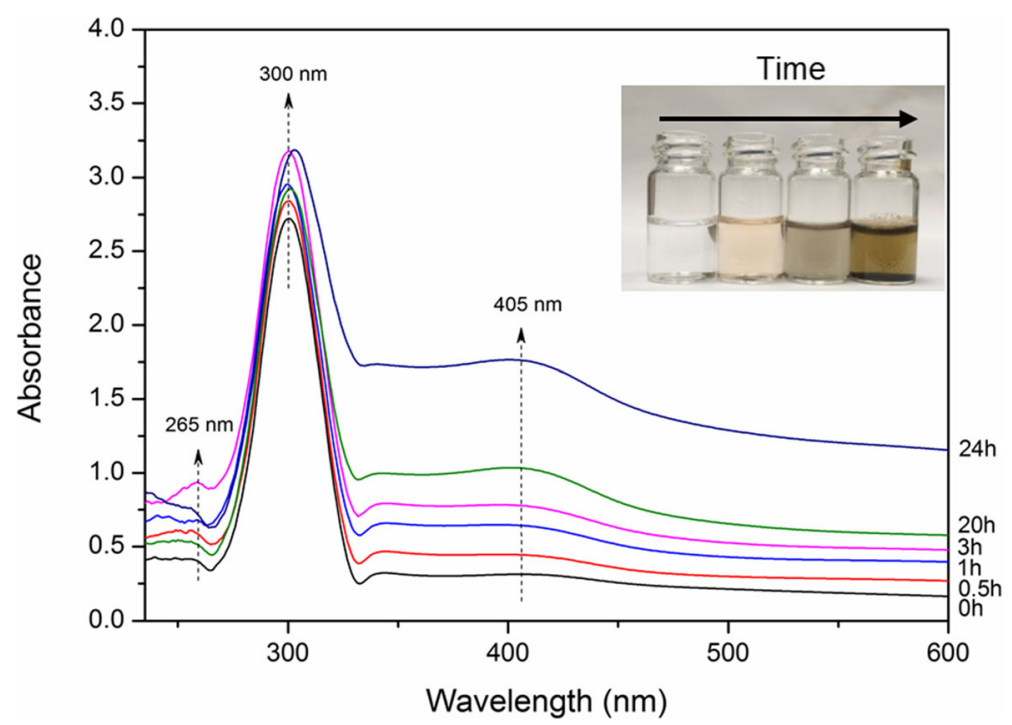

Fig. 3 UV-visible spectroscopy results of PDA polymerisation process from $0 \mathrm{~h}$ to $24 \mathrm{~h}$; the inset shows the colour changing of the PDA solution over time

For untreated aramid fibre, three peaks were exhibited in Fig. 4a, indicating $\mathrm{C}=\mathrm{C} / \mathrm{C}-\mathrm{C}$ in aromatic rings $(284.6 \mathrm{eV}), \mathrm{C}-\mathrm{N}$ group $(286.4 \mathrm{eV})$ and $\mathrm{C}=\mathrm{O}$ group $(288.8 \mathrm{eV})$, respectively. The PDA treated aramid fibre, three new peaks emerged as shown in Fig. 4b, representing the existence of $\mathrm{O}-\mathrm{C}=\mathrm{O}(289.1 \mathrm{eV}), \mathrm{C}-\mathrm{O}(286.5 \mathrm{eV})$ and $\pi-\pi^{*}(290.5 \mathrm{eV})$. These three new peaks demonstrate the successful formation of PDA layer on the surface of aramid fibres. The ratio for oxygen-containing groups increase from 6.35 to $17.50 \%$ while the ratio for $\mathrm{C}=\mathrm{C} / \mathrm{C}-\mathrm{C}$ group decreases from 78.91 to $55.14 \%$ after the PDA treatment. This is mainly attributed to the fact that the intramolecular bonding (hydrogen bonding) in aramid fibre is destructed which activates the originally inert fibre surface. For GO-PDA treated-aramid fibre, its XPS C1s corelevel spectrum, shown in Fig. 4c displays six peak components with binding energies at about 284.6, 288.8, 286.3, 285.3, 287.4, and $290.1 \mathrm{eV}$ respectively, associating to the $\mathrm{sp}^{2}$-hybridized carbon, $\mathrm{O}-\mathrm{C}=\mathrm{O}, \mathrm{C}-\mathrm{O}, \mathrm{C}-\mathrm{N}, \mathrm{C}=\mathrm{O}$ and shake-up satellite $\left(\pi-\pi^{*}\right)$ peak accordingly. Compared with the PDA treated aramid fibre, the ratio for $\mathrm{C}=\mathrm{O}$ group increases from 3.37 to $4.16 \%$. The increasing number of $\mathrm{C}=\mathrm{O}$ group indicates potential hydrogen-bonding interaction with $\mathrm{GO}$ and PDA. The $\pi-\pi^{*}$ bonding also increases from 0.15 to $0.58 \%$. The $\pi-\pi$ stacking is a kind of van der Waals force relating to the molecules' $\pi$-electron backbone system, to form a stable hybrid structure [9]. The aramid fibres are consist of aromatic molecules, which are proven effective for intermolecular $\pi-\pi$ stacking through self-assembly processing $[10,11]$. The

Table 1 Results of the fitting of C1s core level spectra of untreated-, PDA- and GO-PDA-aramid fibre

\begin{tabular}{lllllll}
\hline \multicolumn{7}{l}{ The ratio for functional groups [\%] } \\
\cline { 2 - 7 } & $\mathrm{C}=\mathrm{C} / \mathrm{C}-\mathrm{C}$ & $\mathrm{O}-\mathrm{C}=\mathrm{O}$ & $\mathrm{C}=\mathrm{O}$ & $\mathrm{C}-\mathrm{O}$ & $\mathrm{C}-\mathrm{N}$ & $\pi-\pi^{*}$ \\
\hline Untreated aramid & 78.91 & - & 6.35 & - & 14.74 & - \\
PDA-aramid & 55.14 & 4.53 & 3.37 & 9.60 & 27.21 & 0.15 \\
GO-PDA-aramid & 51.67 & 5.84 & 4.16 & 12.70 & 25.05 & 0.58 \\
\hline
\end{tabular}



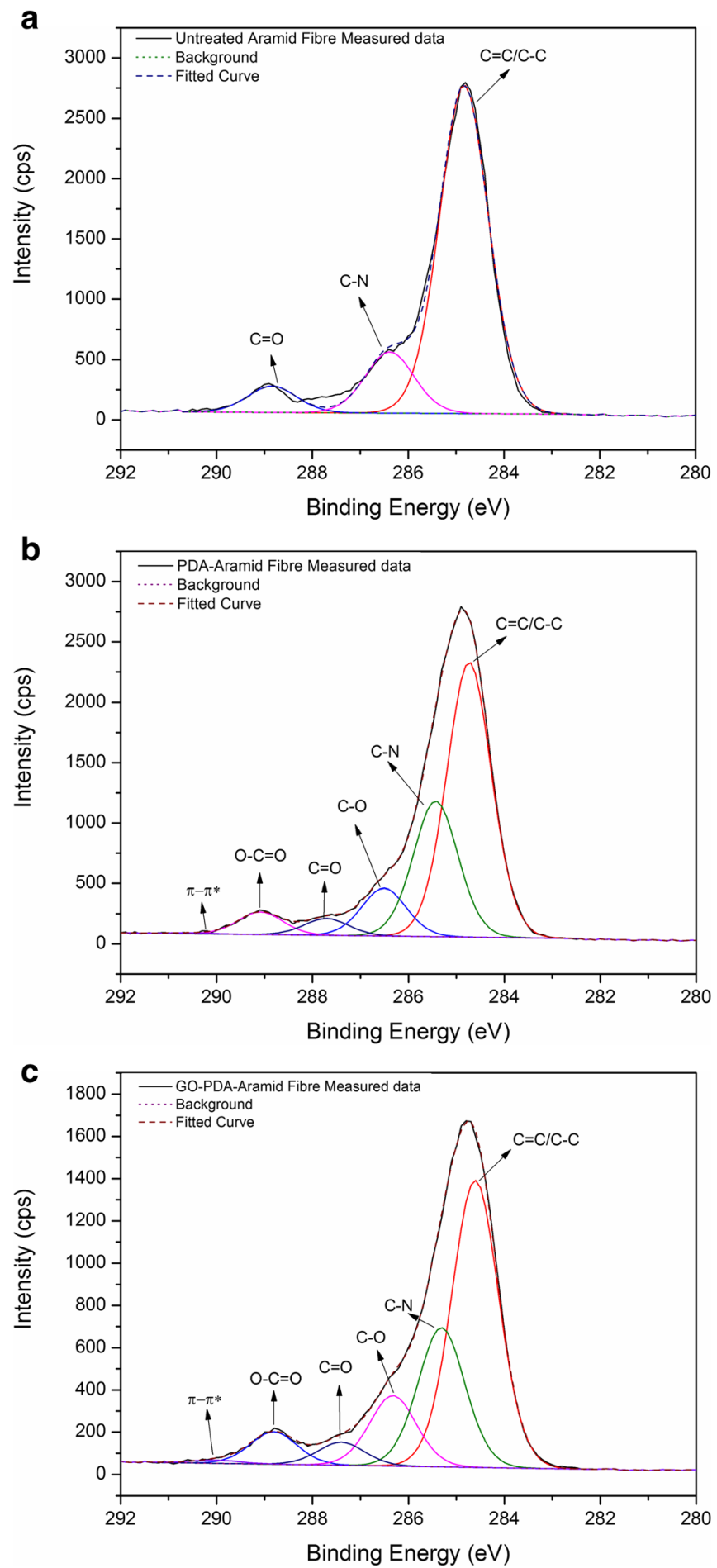

Fig. 4 XPS spectra of C1s of (a) untreated aramid fibre, (b) PDA-aramid fibre and (c) GO-PDA-aramid fibre 
strong $\pi-\pi$ stacking between the graphene oxide surface and the aromatic molecules usually makes the surface stacking quite stable against rinsing or other solution processing. It became evident that the GO-PDA treatment increased the surface activation of aramid fibre and thus increasing their interfacial adhesion strength.

\subsection{Surface Topography of Aramid Fibre}

The surface morphology of aramid fibres before and after the treatment was studied using SEM. It can be seen in Fig. 5a, b that the pristine aramid fibres display a clean and smooth surface. After modification by polydopamine, a homogeneous PDA layer was successfully coated onto the aramid fibre. The PDA-aramid fibres showed an uneven surface with micropits, as shown in Fig. 5c,d. Figure 5e, f indicated that, the roughness of fibre surfaces was further increased by depositing GO onto the PDA coated aramid fibres. The typical thin paper-like wrinkled graphene oxide morphology was observed on the aramid fibre surfaces.
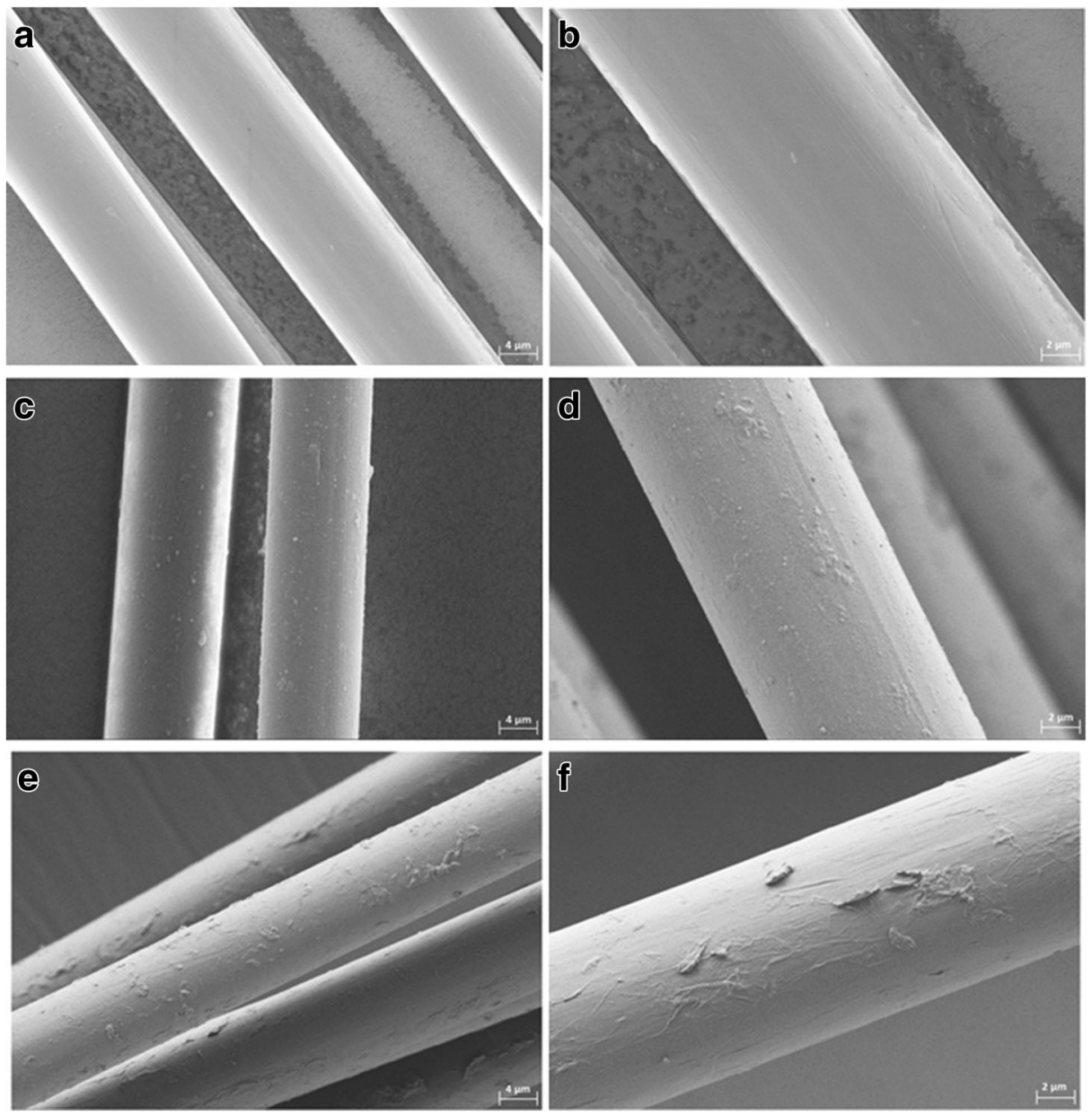

Fig. 5 SEM images of (a) (b) untreated aramid fibres; (c) (d) PDA treated aramid fibres; (e) (f) GO and PDA treated aramid fibres 
The SEM images demonstrated uniform deposition of PDA and GO on aramid fibres, and both enhanced the roughness of the fibre surfaces.

\subsection{The Fibre-Resin Interfacial Shear Strength Analysis}

The interfacial shear strength (IFSS) between aramid fibre and resin was investigated by pulling a fibre out of resin droplet through a knife-edge and the results are shown in Fig. 6. Compared with the untreated aramid fibre composites, the IFSS for the PDA-aramid composites increased from 1.9 MPa to 4.2 MPa by, representing a $121 \%$ increase, and for the GOPDA-aramid composites, the IFSS was increased by $210 \%$ compared to that of untreated aramid fibre composites as shown in Fig. 6. The IFSS increase can be attributed to the increased roughness of the fibre surface caused by PDA treatment and GO-PDA treatment. In addition, the successful grafting of PDA and GO introduced chemical groups which can also enhance the surface reactivity and polarity. The results revealed that the modification applied to the aramid fibre surface by polydopamine and graphene oxide effectively enhances the interfacial property of aramid fibre/epoxy composites.

\subsection{Tensile Strength of Aramid Fibres}

Some research literatures reported fibre surface etching when modified chemically or physically, causing decrease in tensile strength $[12,13]$. To investigate the tensile strength of unmodified and PDA- and GO-PDA- modified aramid fibres, the single fibre tensile test was conducted. As shown in Fig. 7, the single fibre tensile strength of untreated aramid fibre was 2099.2 MPa. For the aramid fibre with PDA deposition, the tensile strength of single fibre was increased to $2470.9 \mathrm{MPa}$. Although only a thin layer of PDA was deposited on the fibre surface, the bulk structure of aramid fibre was protected. After adding GO onto the PDA

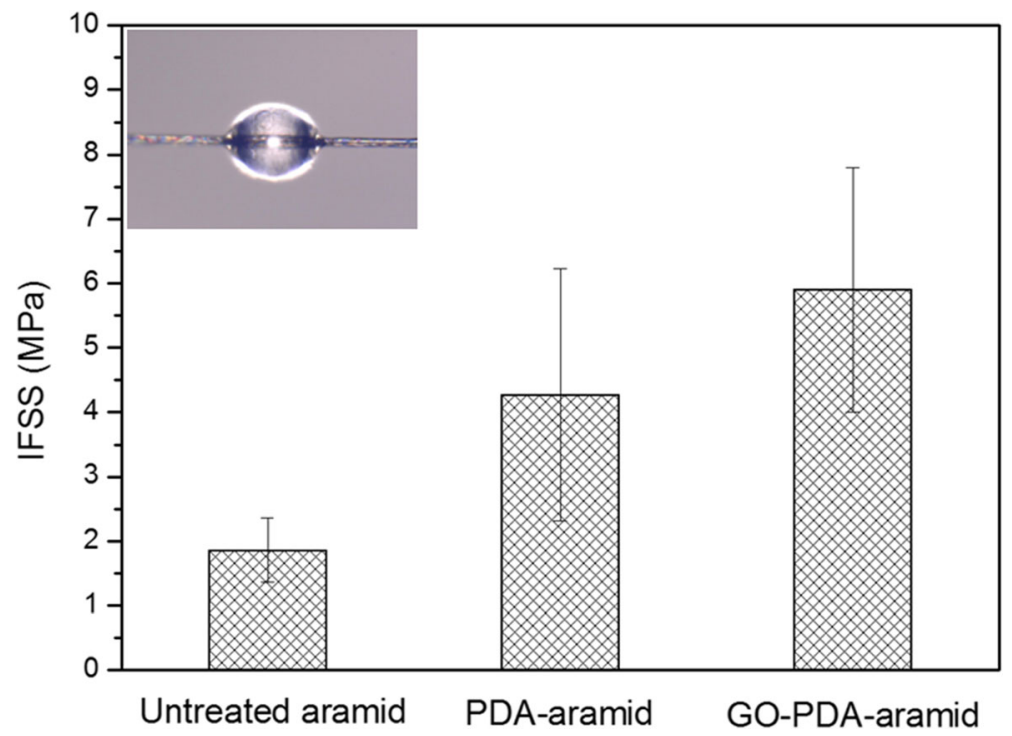

Fig. 6 Interfacial shear strength between single aramid fibre and resin; the inset shows an optical microscope photo of a microbond test specimen 


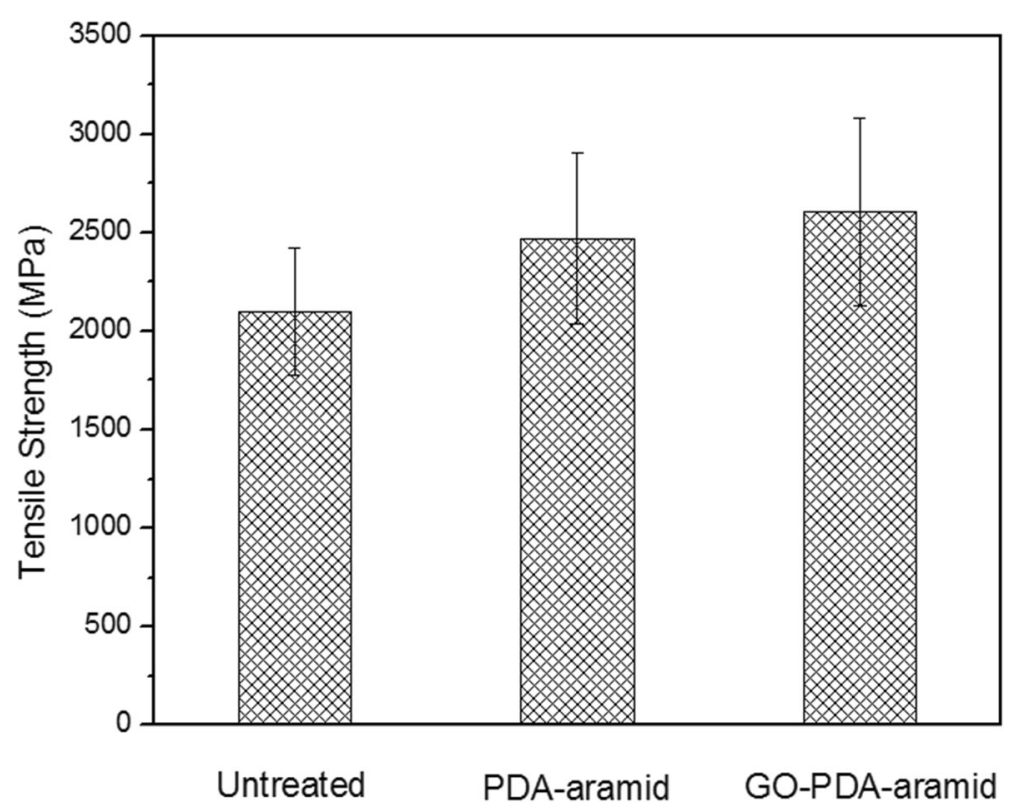

Fig. 7 Single fibre tensile strength of unmodified, PDA- and GO-PDA- modified aramid fibres

deposited fibre surface the single fibre tensile strength became $2603.4 \mathrm{MPa}$, which is $24 \%$ higher than the unmodified aramid fibre. The reason for this increase is believed to be that the PDA and the PDA-GO coatings may have filled up the flaws on the surface of the untreated fibre. This method modified the fibre surface by coating an ultra-thin layer without etching or damaging the bulk structure of the fibre.

\section{Conclusion}

In this study, the GO and bioinspired PDA were used to functionalize aramid fibre, aiming to improve the interfacial adhesion between aramid fibre and epoxy matrix. SEM and XPS confirmed that the aramid fibres were successfully coated by PDA and GO-PDA. XPS demonstrated that more polar functional groups were introduced onto aramid fibre after the treatments, which could affect the fibre surface activation. Besides, the strong $\pi-\pi$ bonding usually makes the surface stacking quite stable against rinsing or other solution processing, which result in an improvement of the interfacial shear strength between fibre and matrix. The SEM observation displayed uniform coating of PDA and GO on the surface of fibre and the modification of GO increased the roughness of fibre surface. As a result, the interfacial shear strength of GO-PDA-aramid fibre composites was improved by $210 \%$. The usefulness of the GO-PDA coating has demonstrated significant increase in IFSS. The surface modification has also protected the bulk structure of fibres without decreasing the tensile strength of single fibre.

This study provides a novel method for surface modification of chemically inert highperformance fibres, which is believed to be beneficial for interface improvement for composites. Furthermore, the polydopamine and graphene oxide grafting will lead to the feasible application of high-performance fibres in the composites industry, aerospace and ballistic protection area. 
Acknowledgements The authors are grateful Bluestar, Chengdu, China for providing the aramid fibres used in this research.

Open Access This article is distributed under the terms of the Creative Commons Attribution 4.0 International License (http://creativecommons.org/licenses/by/4.0/), which permits unrestricted use, distribution, and reproduction in any medium, provided you give appropriate credit to the original author(s) and the source, provide a link to the Creative Commons license, and indicate if changes were made.

\section{References}

1. Ni, N., Wen, Y., He, D., Yi, X., Wang, C., Xu, Y.: Synergistic reinforcement effect of aramid nonwoven fabrics and PVDF on mechanical and damping properties of bismaleimide matrix composites. Compos. A: Appl. Sci. Manuf. 79, 176-182 (2015)

2. George, J., Sreekala, M., Thomas, S.: A review on interface modification and characterization of natural fiber reinforced plastic composites. Polym. Eng. Sci. 41(9), 1471-1485 (2001)

3. Rong, H., Dahmen, K.-H., Garmestani, H., Yu, M., Jacob, K.I.: Comparison of chemical vapor deposition and chemical grafting for improving the mechanical properties of carbon fiber/epoxy composites with multiwall carbon nanotubes. J. Mater. Sci. 48(14), 4834-4842 (2013)

4. Sarmadi M.: Advantages and disadvantages of plasma treatment of textile materials. 21st International Symposium on Plasma Chemistry (ISPC 21), Sunday (2013)

5. Xing, L., Liu, L., Xie, F., Huang, Y.: Mutual irradiation grafting on indigenous aramid fiber-3 in diethanolamine and epichlorohydrin and its effect on interfacially reinforced epoxy composite. Appl. Surf. Sci. 375, 65-73 (2016)

6. Lee, H., Dellatore, S.M., Miller, W.M., Messersmith, P.B.: Mussel-inspired surface chemistry for multifunctional coatings. Science. 318(5849), 426-430 (2007)

7. Wei, Q., Zhang, F., Li, J., Li, B., Zhao, C.: Oxidant-induced dopamine polymerization for multifunctional coatings. Polym. Chem. 1(9), 1430-1433 (2010)

8. Sun, P., Wang, J., Yao, X., Peng, Y., Tu, X., Du, P., et al.: Facile preparation of mussel-inspired polyurethane hydrogel and its rapid curing behavior. ACS Appl. Mater. Interfaces. 6(15), 12495-12504 (2014)

9. Zhang, Z., Huang, H., Yang, X., Zang, L.: Tailoring electronic properties of graphene by $\pi-\pi$ stacking with aromatic molecules. J. Phys. Chem. Lett. 2(22), 2897-2905 (2011)

10. Gao, W., Kahn, A.: Controlled p-doping of zinc phthalocyanine by coevaporation with tetrafluorotetracyanoquinodimethane: a direct and inverse photoemission study. Appl. Phys. Lett. 79(24), 4040-4042 (2001)

11. Zang, L., Che, Y., Moore, J.S.: One-dimensional self-assembly of planar $\pi$-conjugated molecules: adaptable building blocks for organic nanodevices. Acc. Chem. Res. 41(12), 1596-1608 (2008)

12. Qian, H., Bismarck, A., Greenhalgh, E.S., Kalinka, G., Shaffer, M.S.: Hierarchical composites reinforced with carbon nanotube grafted fibers: the potential assessed at the single fiber level. Chem. Mater. 20(5), 1862-1869 (2008)

13. Jia, C., Chen, P., Wang, Q., Wang, J., Xiong, X., Ma, K.: The effect of atmospheric-pressure air plasma discharge power on adhesive properties of aramid fibers. Polym. Compos. 37(2), 620-626 (2016) 